\title{
Learning how to reverse early enamel erosion
}

The three Cs - causes, consequences and care - of early enamel erosion are being explored at the British Dental Conference and Dentistry Show (BDCDS) (17-18 May 2019) by Unilever, the makers of the Regenerate ${ }^{\mathrm{TM}}$ Advanced Enamel Serum and Toothpaste.

Key members of Unilever's Research and Development team will be giving bite-sized 20 minute lectures twice an hour, every hour, on stand N70. Attendance will be recognised with CPD certification as well as a free trial of the Regenerate regime

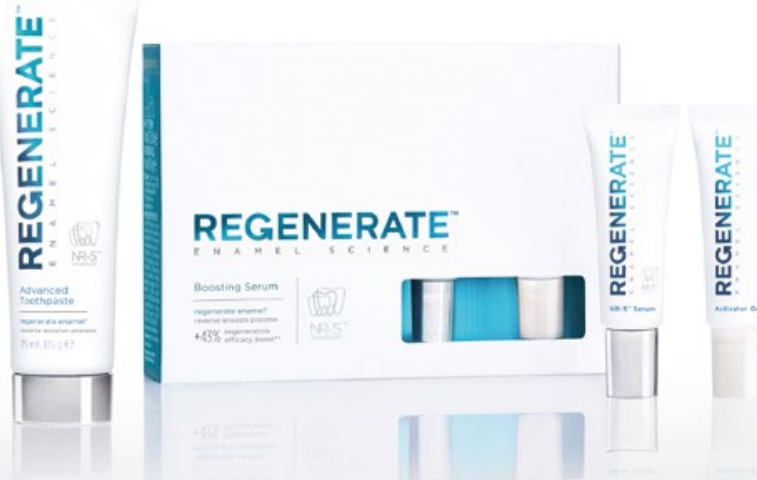

Visit Stand N70 at the Dentistry Show to learn more about the Regenerate range

consisting of Advanced Toothpaste and Advanced Enamel Serum.

Until recently, there has been little in the dental professionals' armoury for patients with the early signs of enamel erosion, once diagnosed. However, scientists at Unilever have changed all that with a remineralisation regime. REGENERATE Enamel Science is the first oral care system with NR-5 technology which regenerates tooth enamel mineral ${ }^{*}$, reversing the early enamel erosion process and so keeping teeth healthy and strong. $80 \%$ of common tooth problems, such as sensitivity, transparency and yellowing, are caused by enamel erosion and acid attacks.

REGENERATE Enamel Science Advanced Toothpaste regenerates enamel mineral and helps to restore natural whiteness while strengthening teeth against cavities.

The Advanced Toothpaste is powered by an exclusive and clinically proven NR-5 technology. The NR-5 ingredients combine to form a fresh supply of tooth enamel mineral which wrap and integrate onto teeth. Thanks to its unique formulation, the REGENERATE Enamel Science toothpaste has distinctive product characteristics: a dense formula with a rich texture due to the high content of ingredients and a fresh mint sensation.

The exclusive Advanced Enamel Serum increases the effectiveness of the Advanced Toothpaste by $43 \%{ }^{* *}$, maximising the power of Enamel Mineral Regeneration*, even in interdental spaces that are hard to reach. The application of the Serum is designed to kick start the process and get to work immediately. It helps to remineralise enamel mineral ${ }^{*}$ as it is clinically proven to form new hydroxyapatite mineral directly onto the enamel surface with identical chemical composition and crystalline structure as natural tooth enamel mineral. ${ }^{1}$ This patented, clinically proven NR-5TM technology provides focused protection for patients' teeth and can reverse the early enamel erosion process. Each Advanced Enamel Serum kit contains one NR-5 Serum and one Activator Gel plus two custom-fit mouth trays and a mixing stick. When combined with the Activator Gel, the NR-5 Serum unleashes a concentrated boost of the formula, helping to reverse the early enamel erosion process. REGENERATE Enamel Science Advanced Enamel Serum should be used every month, for three consecutive days, preferably before bed time. For best results, use it in combination with the daily Advanced Toothpaste.

\section{Public awareness}

Growing professional awareness about the consequences and treatment of enamel erosion is not spilling over into public awareness. An Oral Health Foundation survey conducted in 2018 sought to find out what the public knew about enamel erosion. Only a fifth of respondents correctly identified that $80 \%$ of common tooth problems including tooth sensitivity, cracking, thinning or yellowing are caused by enamel erosion. ${ }^{2}$

Another study conducted on behalf of Unilever showed that erosive tooth wear is a common and increasing prevalent problem and that as many as 55\% of young people aged between $18-35$ in the UK are affected. ${ }^{3}$ Stress-related grinding is only one cause. Enamel erosive wear is also a cumulative process triggered by repeated acid attacks, and the steep increase in people experiencing it has been attributed to a rise in people consuming fizzy drinks, sports drinks, and juices, vinegar based dressings, wines and cocktails.

A visit to stand N70 at the BDCDS will allow dental professionals the opportunity to learn about ways to enlighten patients, as well as diagnose their early enamel erosion. For more information please visit www.regeneratenr5.co.uk.

\section{References}

1. Sun $Y, L i X$, Deng $Y$ et al. Mode of action studies on the formation of enamel minerals from a novel toothpaste containing calcium silicate and sodium phosphate salts. J Dent 2014; 42: S39-S45.

2. Oral Health Foundation. Oral Health Foundation Survey. 2018.

3. Bartlett D W, Lussi A, West N X et al. Prevalence of tooth wear on buccal and lingual surfaces and possible risk factors in young European adults. J Dent 2013; 41: 1007-1013.

"Regenerate Enamel Science serum and toothpaste act on early invisible signs of enamel erosion. Helps to regenerate enamel by restoring its mineral content and micro-hardness with regular use. Clinically proven.

"As demonstrated in a three day in vitro test measuring enamel micro hardness:Combined use of toothpaste and serum vs. toothpaste only. 